\title{
Impact of Flow Based Market Coupling on the European Electricity Markets
}

\author{
Rafael Finck ${ }^{1}$
}

Received: 4 April 2021 / Revised: 12 October 2021 / Accepted: 29 October 2021 / Published online: 18 November 2021

(c) The Author(s) 2021

\begin{abstract}
Flow Based Market Coupling is the target model for determining exchange capacities in the internal European Electricity Market. It has been in operation in Central Western Europe since 2015 and is scheduled to be extended to the wider Core region in the near future. Exchange capacities have a significant impact on market prices, exchanges and the energy mix, thus also determining the $\mathrm{CO} 2$ footprint of electricity generation in the system. Stakeholders therefore need to develop an understanding for the impact of Flow Based Market Coupling and the parameter choice, like the minimum exchange capacities introduced in 2020, on the market outcome. This article presents a framework to model Flow Based Market Coupling and analyse the impact of different levels of regulatory induced minimum trading capacities as well as the effect of the extension towards the Core region. Electricity prices, exchange positions and the number and nature of binding constraints in the market results under different market coupling scenarios are investigated. The results show that increased level of minimum trading capacities in CWE market coupling decrease the German net export position by up to 7TWh or $23 \%$, while French exports increase by up to $10 \mathrm{TWh}$ or $9 \%$. The different transfer capacity in the scenarios induce a price difference of up to $13 \%$. Increased exchange capacities allow for more base load generation with the corresponding effects for the $\mathrm{CO} 2$ emissions of the system. The nature of coupling constraints is highly dynamic and dependent on the system state, which makes the suitability of static NTC values in energy system scenarios questionable.
\end{abstract}

Keywords Flow Based Market Coupling $\cdot$ Remaining available margin $\cdot$ Zonal pricing $\cdot$ Electricity grid $\cdot$ Electricity market $\cdot$ Congestion management

\section{Introduction}

Electricity wholesale prices are the basis for many analyses in energy economics, where cost of electricity impacts investment decisions in generation and demand technologies. Market results also determine the emissions of the energy sector as well as the degree to which renewable energy systems can economically feasibly be integrated into the energy system. For many detailed studies with reduced spatial scope, prices and emissions of "electricity from the grid" play a crucial role, be it in the context of energy autonomous municipalities, energy concepts for districts, diffusion scenarios of PV battery storage systems or elec-

Rafael Finck

rafael.finck@kit.edu

1 Chair of Energy Economics, Institute for Industrial Production (IIP), Karlsruhe Institute of Technology (KIT), Karlsruhe, Germany tric mobility. The price and generation technology for the majority of procured electricity in Europe is determined on the Day-Ahead market, by the Single Day-Ahead Coupling (SDAC). After Bulgaria joined on 9 March 2021, SDAC covers about $95 \%$ (1,500 TWh/year) of the EU's consumption (ENTSO-E (2021a)).

Market Coupling and the respective determination of available transfer capacities have a large impact on the outcome of electricity markets. It allows for the efficient utilization of physical transport capacities and increases liquidity in markets (Next Kraftwerke (2021)), impacting energy mix, fuel consumption and $\mathrm{CO} 2$ emissions in the coupled market zones. In large parts, the market coupling between price zones is based on bilateral exchange capacities, so-called Net Transfer Capacities (NTC), which are also widely applied scenario-based energy system analyses. While easy to apply, NTCs cannot account for the physical reality in meshed electricity networks, because they do not reflect how exchanges affect each other. This is due to the fact, that power flows follow the physical 
parameters of the network and only partly match commercial exchanges. To overcome these limitations, the Flow Based Market Coupling (FBMC) methodology was proposed and implemented in the Central Western Europe (CWE) region in May 2015, to explicitly consider grid constraints in the process. FBMC is the target model for the internal electricity market and is planned to be introduced in the larger Core region in 2021, then covering the borders of the bidding zones Austria, Belgium, Croatia, Czech Republic, France, Germany/Luxembourg, Hungary, the Netherlands, Poland, Romania, Slovenia and Slovakia (ENTSO-E (2020a)). Apart from desirably allowing for larger exchanges compared to NTC market coupling, the flow based (FB) methodology also allows for a more transparent representation of market coupling constraints, which makes it possible to attribute exchange limitations to single grid elements (Marjanovic et al (2018)).

Unfortunately, these advantages come at a price and result in largely increased input data sets covering the transmission grid topology and parameters, spatially resolved generation units, the ability to produce regionalized capacity allocations and output for new-built power plants (especially decentralized renewable energy sources (RES)), which are necessary for the adequate simulation and analysis of present and future energy systems. For market modellers and practitioners alike, it is important to develop an understanding for the implications FBMC has on the European Electricity Market. In particular, to assess the difference between the application of FBMC and the wellknown NTC constraints, with respect to the most relevant scenario outcomes like electricity prices, exchange positions and emissions resulting from the energy mix.

In this article, a framework, which enables this evaluation, is presented and the effects of different market coupling approaches are quantified in real-world scenarios for the inter-connected European electricity market. Section 2.1 revisits the main concepts of FBMC to facilitate the reader's appreciation of the following literature review on FBMC in Sect. 2.2. The developed model and the analysed scenarios are presented in Sect. 3. Section 4, covers the main findings and assesses the magnitude of change for market outcomes for the year 2025 using NTC and FB constraints. Moreover, the impact of minimum exchange capacities (minRAM) introduced in 2020 on the outcome of FBMC are investigated. In Sect. 5, the limitations of the presented approach and wider implications of the results are discussed. Section 6 concludes and gives an outlook on future research.

\section{Flow based Market Coupling}

FBMC was introduced in the CWE region in 2015 to better account for the physical electricity network constraints in the market clearing procedure. The goal is to increase cross-border capacities, promote supplier competition, increase grid security and minimize prices across in the market zones (Next Kraftwerke (2021)). This Section revisits methodology and important concepts of FBMC to provide the reader with context for the following literature review and the remainder of the article.

\subsection{Methodology}

For the calculation of FBMC the information gap, which arises from the zonal market design in the European electricity markets has to be closed. Due to the separation of electricity suppliers and grid operators accompanied by the clearing of anonymous bids at the energy exchange, the grid operators cannot know which power plants participate in additional commercial exchange and thus how much the grid infrastructure is loaded. For the necessary calculation of available transmission capacities, the grid operators have to approximate the market outcome to forecast the grid utilization. This approximation is called the base case, which consists of the forecasted demand, supply, exchange position and grid topology.

In the European market design, bidding zones are regarded as (almost) free of congestion. This limits the grid elements, which are considered in the market clearing to constrain commercial exchanges. The relevant elements are identified with linearized sensitivity factors called Power Transfer Distribution Factors (PTDF). Only lines and transformers, which are affected by cross-border exchanges above a certain threshold (often 5 percent, e.g. CREG (2017)) are included. This selection threshold is referred to as PTDF threshold for the remainder of the article. Because the market outcome has to result in a secure grid operation, possible grid outages, which reduce the available capacity on the lines, are considered. Only a limited number of contingencies have a critical impact on the grid, threatening security. Contingency screening is performed to identify the most relevant outages, which are then included as additional constraints. The relevant grid elements are called Critical Network Elements under a Contingency (CNEC). In this article, outage constraints are included in the FBMC if the line loading in the outage simulation lies above a certain threshold referred to as CNEC threshold.

Without the information which bid at the energy exchange is linked to which generator and thus unable to exactly determine the effect of commercial exchanges on the network elements, the grid operators have to develop strategies to approximate the impact of trade results on the grid. This is accomplished by assigning linear participation factors to generators, which distribute the delta in net export position (NP) of a market zone to the generators most 
Table 1 Literature overview on articles covering FBMC

\begin{tabular}{ll}
\hline Reference & Scope and Conclusion \\
\hline Kurzidem (2010) & \multicolumn{1}{c}{ Category I: Conceptual work on stylized examples or reduced temporal resolution } \\
Mekonnen and Bel- & $\begin{array}{l}\text { Analysis of market power in zonal power markets with FBMC constraints } \\
\text { mans (2012) }\end{array}$ \\
Marien et al (2013) & $\begin{array}{l}\text { stylized 3-zone example system } \\
\text { Analyse the impacts of Generation Shift Key strategy and Flow Reliability Margin on the outcome of FBMC in a } \\
\text { stylized system representing CWE with changing zonal configurations. Smaller zones reduce the uncertainty of Flow } \\
\text { Reliability Margin. Generation Shift Keys should reflect best available forecast }\end{array}$ \\
Schavemaker and & $\begin{array}{l}\text { Compare FBMC and NTC market coupling in a stylized system. FBMC generally offers superior competition for } \\
\text { scarce capacity compared to NTC market coupling. Scarcity of transmission capacity has to be represented by zones } \\
\text { to be subject to market allocation }\end{array}$
\end{tabular}

Sores et al (2013) Present a FBMC model for Czech republic, Slovakia and Hungary, which is evaluated for the first week of 2012. Complex bids are included in a MIQP formulation

Hagspiel et al (2014) Present a framework for power system extension considering FBMC. The approach is tested in a 3-node system and then extended to a 200-bus representation of the European transmission system

Bjorndal et al (2018) Analyse FBMC on stylized 3, 6 and 24 bus grids for single hours. The identified increased social welfare in the market results comes at the cost of higher re-dispatch

Felten et al (2019) The basic concepts of FBMC and sensitivities of certain parameter variations are analysed on a stylized 4-node example. A framework for FBMC in CWE region is presented

Byers and Hug (2020) Compare different approaches for the base case, re-dispatch and market clearing on a 3-zone system for one month. The base case resembling the nodal result leads to lowest system cost

Lang et al (2020) Present an approach for integrating grid constraints in the spot market evaluated on a stylized central European region (29 nodes) labelled FBMC. The article highlights the importance to integrate FBMC in market and grid analyses instead of NTC market coupling to have a more adequate representation of physical constraints

Poplavskaya et al Propose the integration of nodal information for some generators in the spot market to relieve critical branches. The (2020)

Schönheit et al (2020) approach is evaluated on a two zone, 6 node system

Felten et al (2021)

Present an open-access model including a test network for experiments with the FB methodology

Compare FBMC to a nodal market clearing in a stylized single hour, 4-node system. Conclude that the closer the approximation of the base case is, the lower the welfare losses by FBMC are, compared to nodal pricing

Henneaux et al (2021) Present a FBMC framework for a 3 area, 96-bus system. In this setup the impact of minimum threshold for crosszonal capacities (minRAM) are investigated. Different re-dispatch schemes and the resulting implications on welfare are analysed

Weinhold and Mieth Formulate a probabilistic FBMC, which includes uncertainty in RES generation in the Flow Reliability Margin in a (2021) 118-bus system. The reduced exchange in the probabilistic Day-Ahead market clearing proves more robust against real-time deviations than deterministic FBMC

\begin{tabular}{|c|c|}
\hline \multicolumn{2}{|r|}{ Category II: Reduced temporal or spatial scope of real-world systems /Analysing specific aspects (mainly in CWE) } \\
\hline $\begin{array}{l}\text { van den Bergh et al } \\
\text { (2016) }\end{array}$ & $\begin{array}{l}\text { Presents the key concepts of FBMC as applied in the CWE and identify challenges with regard to the transparency of } \\
\text { the process }\end{array}$ \\
\hline Dierstein (2017) & $\begin{array}{l}\text { Analyses the impact of different Generation Shift Key Strategies on the market outcome for the CWE region in } 16 \\
\text { time steps. The different strategies have significant impact on line flows; however, the reduced temporal resolution } \\
\text { limits the possibility to generalize results }\end{array}$ \\
\hline Finck et al (2018) & $\begin{array}{l}\text { Compare the impact of six different Generation Shift Key strategies on market outcome in CEE region for a scenario } \\
\text { in } 2020 \text {. Results show that the difference compared to NTC market coupling is larger than the impact the GSK strate- } \\
\text { gies have on the energy mix in the investigated zones }\end{array}$ \\
\hline Sebestyen et al (2018) & $\begin{array}{l}\text { Analyse the impact of three Generation Shift Key strategies in the grid of Belgium and highlight the possible impacts } \\
\text { on the FB domain }\end{array}$ \\
\hline Felling et al (2019) & $\begin{array}{l}\text { Price zone configuration for market coupling in CWE and Switzerland is investigated under FBMC. Improved bidding } \\
\text { zones can reduce re-dispatch needs by over } 90 \% \text {. New zones reduce producer rents in Germany and outside CWE, } \\
\text { while increasing it in France }\end{array}$ \\
\hline Matthes et al (2019) & $\begin{array}{l}\text { Compare different level of minimum capacitites in the CWE region on market results and resulting congestions in the } \\
\text { grid. Although increased minimum capacities increase welfare in the market results, the FB results no longer repre- } \\
\text { sents physical reality in the grid making additional remedial actions necessary }\end{array}$ \\
\hline Bo et al (2020) & $\begin{array}{l}\text { FBMC is applied in the Nordic area with exogenous FB parameters from } 2017 \text { for scenario years } 2020,2022 \text { and } \\
2022 \text { with increased wind generation. Price differences between zones are reduced and FBMC allows Nordic to export } \\
\text { more energy }\end{array}$ \\
\hline
\end{tabular}


Table 1 (Continued)

Kristiansen (2020) Qualitative comparison of FB and NTC market coupling. Summary of historic parallel runs and implications for practitioners. Highlights the difficulties to understand and replicate FBMC for traders as well as the necessity to develop models, which are capable of replicating FB market fundamentals in the medium/long-term

Makrygiorgou et al (2020)

Compare NTC and FB market coupling for several zonal combinations on South Eastern Europe utilizing the Common Grid Model of this region. A single day is analysed considering the inter-connectors as critical branches. Larger trading capacities are offered under FBMC than NTC market coupling

Schönheit et al (2021a)

Analyse three levels of minRAM in the CWE region in combination with three different GSK strategies. Due to computational burden, only two weeks of 2016 are analysed. For all analysed scenarios, the welfare gains in the market are more than offset by losses in the congestion management. The unequal share of welfare effects between consumers and producers is highlighted. Largest welfare effects are reported for increased minRAM from $20 \%$ to $45 \%$

Schönheit et al (2021b)

Historic CNECs are matched to model data and additional constraints added to the problem to resemble historic flows. Afterwards, different GSKs are evaluated for FB market coupling for one week in 2018 for CWE FBMC. The potential beneficial effect of GSKs on the size of the FB domain is highlighted with the remark that for the welfare evaluation redispatch cost have to be included in the analysis

Weinhold (2021) Constructs a 2020 and 2030 scenario for CWE based on open data and compare different minimum trading capacity levels (minRAM) with regard to market effects and welfare including congestion management

Zad et al (2021) Propose a clustering approach for FB domains to incorporate FBMC into adequacy studies

Category III: Frameworks for comprehensive system analysis considering FBMC

Carlini et al (2020)

Present a detailed FBMC model for the Italy North Capacity Calculation Region for the year 2017 with real-world grid data. The approach resembles CWE FBMC. Results show higher import and lower prices in Italy compared to NTC market coupling

Matthes et al (2017) Present a framework to include security constraints efficiently into FBMC in large power systems. Additionally, a linearization of Generation Shift Keys over time is proposed to reduce computational complexity

Marjanovic et al (2018) Investigate the effect of an extended FB region (adding Denmark West, Poland, Czech Republic, Hungary, Slovenia, Slovakia and Italy North to CWE).The NTCs in the scenario lead to larger re-dispatch needs than FB results, while the extended FB region increases price convergence and results in a slight shift in net positions

Wyrwoll et al (2018) Propose a FBMC framework and evaluate it in a 2025 scenario for an extended Core region without the consideration of contingencies

Wyrwoll et al (2019) Analyse the impact of different base case methods on the market outcome for FBMC at the German borders (except Switzerland). An NTC approach performs better, if NTCs are appropriately selected, while power flow analysis at zero net positions allows incorporating the explicit grid expansion state. Due to an applied minimum capacity, results do not differ much

likely participating in this change. These factors are called Generation Shift Keys (GSK).

Having determined the relevant grid elements to incorporate in the market coupling and having forecasted the effects of a change in net position on the power flows, the grid operators have to determine the Remaining Available Margin (RAM) on these grid elements, which can be used for commercial exchanges. Due to the meshed nature of the European transmission grid, even in situations with balanced net positions, CNECs are loaded to a certain degree. The RAM is the remaining capacity, deducting the physical transmission capacity by loop and transit flows and a security factor to account for uncertainties in the FB calculation process (Flow Reliability Margin).

Within the Clean Energy Package (European Commission (2019)), the regulator has introduced a minimum share of physical capacities, which need to be made available for commercial exchange, the so-called minRAM. These minimum capacities were introduced in 2020, but allowed for derogations so they only come into full use in the year 2025 .

\subsection{State of Research}

The body of literature on FBMC has been growing in recent years and is summarized in Table 1 . It can be classified into three groups with regard to the scope of the analysis all of which provide important insights into different aspects of FBMC, which are presented in the following.

The first group incorporates mainly conceptual work regarding FBMC (Kurzidem (2010), Mekonnen and Belmans (2012), Marien et al (2013), Schavemaker and Beune (2013), Sores et al (2013), Hagspiel et al (2014), Lang et al (2020), Poplavskaya et al (2020)) and fundamental research of parameter choice like base case calculation (Byers and Hug (2020), Felten et al (2021), minimum capacities (Henneaux et al (2021)) or Flow Reliability Margin (Weinhold (2021)) in the FBMC methodology. The research is mainly performed on stylized systems or with reduced spatial or temporal resolution, but provides important insights on what to keep in mind when applying FBMC to real life scenarios. While qualitative conclusion can be derived from the results, quantitative interpretation remains difficult due to the difference to real-world systems. 
Fig. 1 Overview of input parameters and model flow
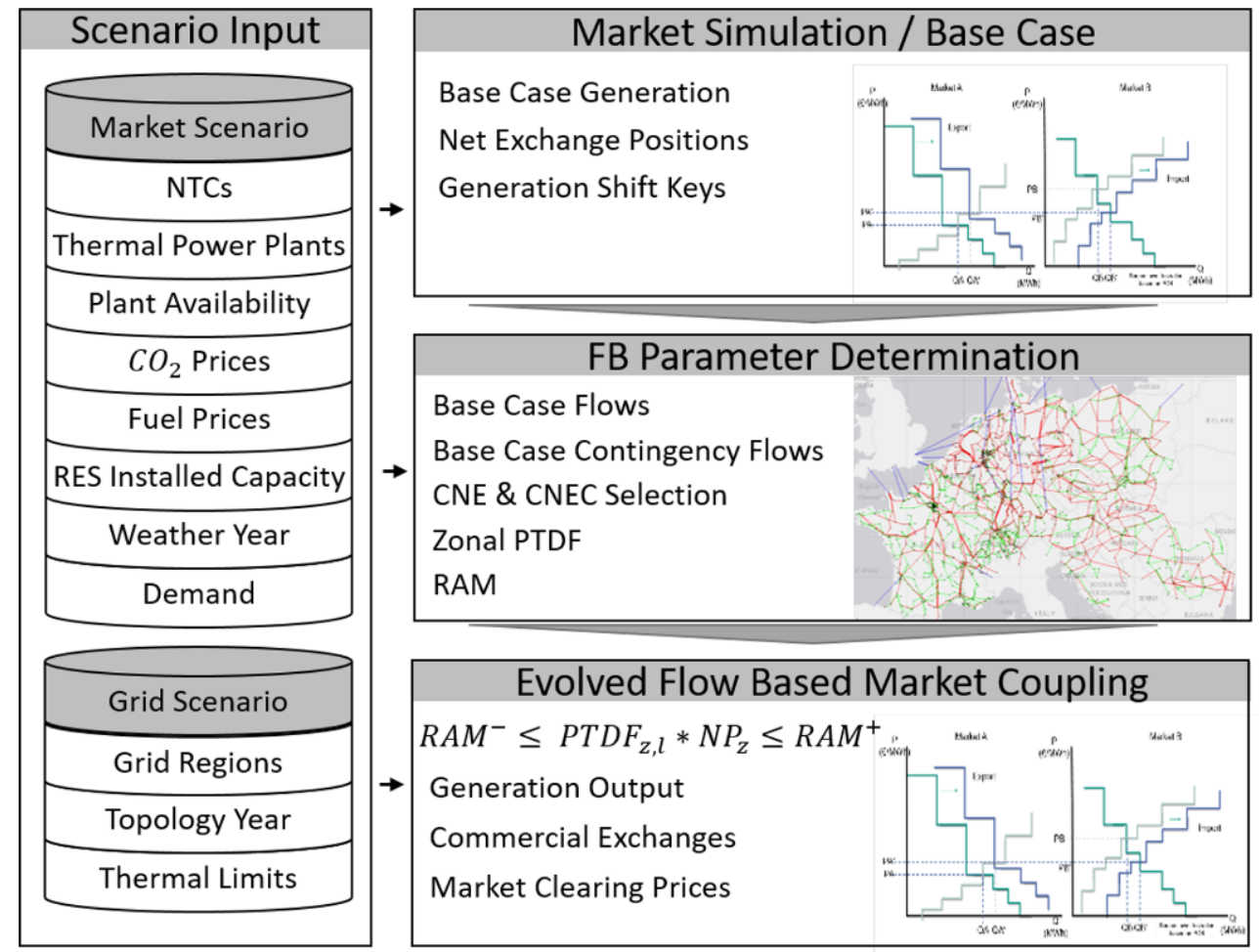

\section{Evolved Flow Based Market Coupling} $R A M^{-} \leq P T D F_{z, l} * N P_{z} \leq R A M^{+}$ Generation Output Commercial Exchanges Market Clearing Prices

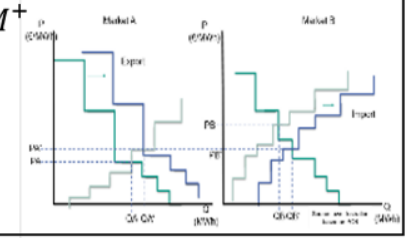

The second group covers analyses of FBMC on realworld systems but with reduced temporal or spatial scope. The majority of research in this group covers the analysis (van den Bergh et al (2016), Kristiansen (2020)) or simulation of (historic) FBMC results in the CWE region (Dierstein (2017), Felling et al (2019), Matthes et al (2019), Schönheit et al (2021a), Schönheit et al (2021b), Weinhold (2021)). Some articles look at different sub-regions, where the effects of FBMC are analysed, e.g. Finck et al (2018), who analyse different GSK strategies in the Central Eastern Europe region, Makrygiorgou et al (2020), who compare FBMC to NTC market coupling in several zonal combinations in South Eastern Europe and Bo et al (2020), who compare FBMC and NTC market coupling in the Nordic region. Zad et al (2021) propose a clustering approach for the flow based domain in the CWE region.

A key insight from groups one and two is that base case and Generation Shift Keys need to match realisation as close as possible for FBMC to have a cost-efficient outcome (e.g. Marien et al (2013). For GSK strategy "pro rate" seems to be a common understanding for good fit (Felten et al (2021), Schönheit et al (2020)). This follows the same idea of a close match of GSK and base case with realisation, hence GSKs based on the specific base case seem reasonable. Some authors raise awareness, that the analysis of cost-effectiveness and welfare impacts should not be limited to the analysis of market results, but need to incorporate the congestion management, which is necessary to ensure secure grid operation (Schönheit et al (2021b), Felten et al (2021)).

The third group of research consists of more comprehensive frameworks, which are capable of analysing the effects of FBMC in full-scale, consistent energy system scenarios, including an explicit grid representation with expansion path, power plant and RES (de-) commissioning with hourly resolution as well as the relevant methodological variety necessary for quantitative analysis of FBMC in the wider European context. Carlini et al (2020) present a detailed FBMC model for the Italy North capacity calculation region and while the real common grid model for this region is used, the framework considers a reduced flow based region not covering the Core region and is limited to historic data. Matthes et al (2017) present a framework to include security constraints into the FBMC methodology efficiently in large-scale power systems but are limited in scope to the CWE region. Marjanovic et al (2018) analyse the effect of an increased flow based region, which includes Italy North and Denmark West but misses Croatia and Romania not resembling the Core capacity region. Wyrwoll et al (2018) cover the Core region but neglect security constraints. Wyrwoll et al (2019) analyses the affect of base case methods in a focus region covering Germany and the electric neighbours. To the best of the author's knowledge, no framework has been presented, which is capable of covering FBMC with an hourly resolution and accounting for security constraints, with a nodal representation of the Core 
Fig. 2 Electricity network model for the Core region for the scenario year 2025

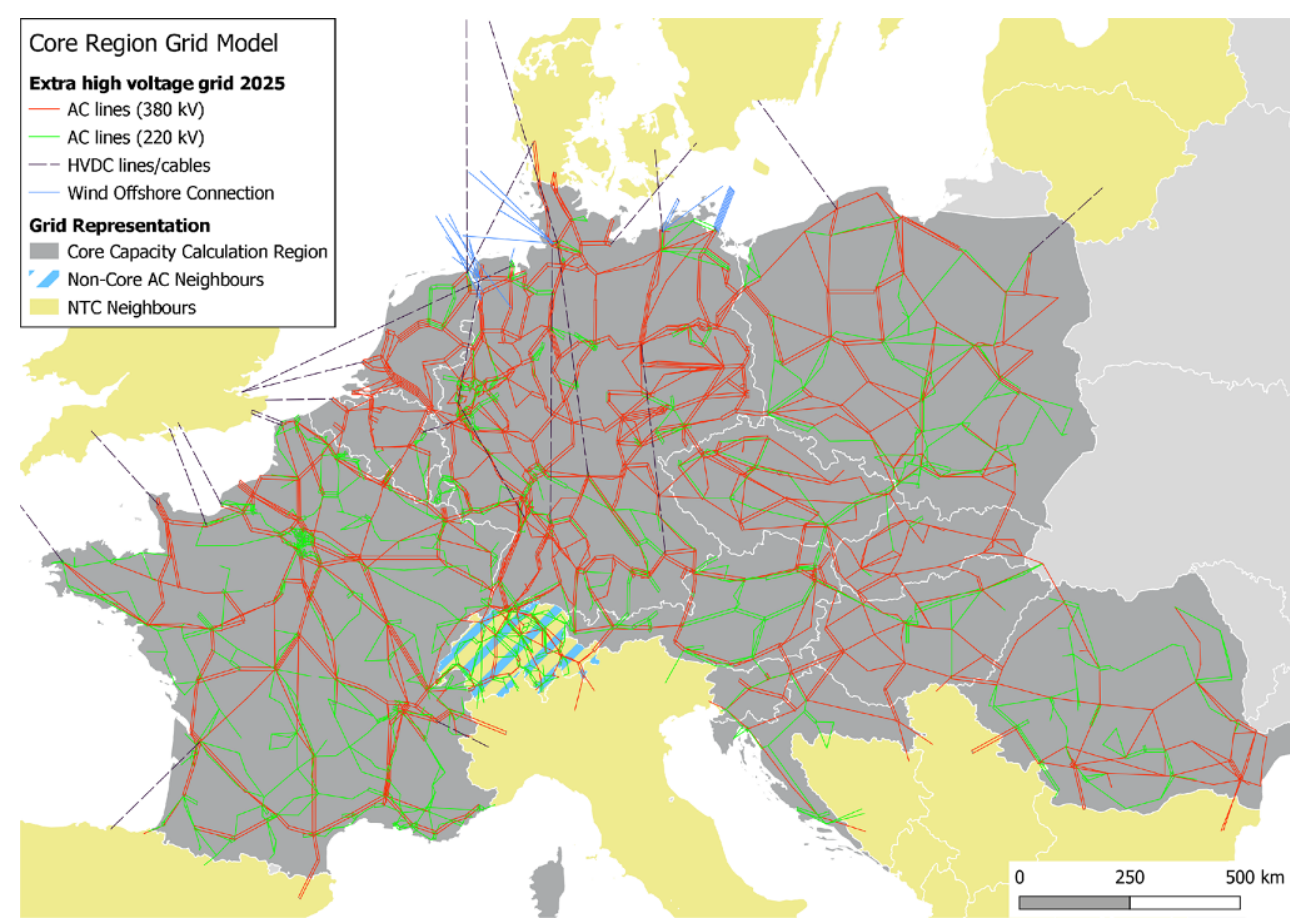

region including consistent scenario information regarding grid expansion, power plant fleet and demand development. Such a framework is necessary to adequately assess quantitative implications of FBMC on the European Electricity Markets in the near future after the extension of the flow based region to the Core capacity calculation region. The article at hand presents a framework capable of these requirements and quantifies the implications of FBMC for several scenarios in the CWE and Core region.

\section{Modelling Framework}

This Section presents the modelling framework, which is used for the analysis. It introduces the modelling scope in Sect. 3.1 and provides information on the input data for the analysed scenarios in Sect. 3.2.

\subsection{Model Description}

The presented model framework consists of a Day-Ahead wholesale market model and a transmission grid model. Both models use the same input data, which are aggregated to bidding zone level for the zonal market simulation. The market model is used for NTC market simulations, which also represent the base case for FBMC, and to solve the market coupling with the derived FB constraints. The model structure is shown in Fig. 1.

The market model covers a unit-wise representation of the European thermal power plant fleet, which is based on
Platts (2016), including the member states of the EU-27, except for Malta, Cyprus and Ireland, extended for Albania, Bosnia and Herzegovina, Great Britain, Kosovo, North Macedonia, Montenegro, Norway, Serbia and Switzerland. For the regionalization of renewable energy sources and demand, the methodology and weather data presented in Slednev et al (2017) and Slednev et al (2018) are applied. The NTC results of the model are used as base case from which power flows on the relevant grid elements are determined in normal operation and contingency cases for relevant outages. Additionally, the GSKs are determined for each grid node according to the generators energy output in the base case, as described in Sect. 2.1.

The grid model is the detailed transmission grid model presented in Ruppert et al (2020), which is extended for the countries to complete the Core region (Croatia, Hungary, Romania, Slovenia, Slovakia) and the high voltage direct current (HVDC) inter-connectors to neighbouring bidding zones. Grid data for these countries are based on ENTSO-E (2021b) and ENTSO-E (2016) including expansion measures from the Ten Year Network Development Plan (TYNDP) 2018 (ENTSO-E (2018)) and national expansion plans, where applicable. The grid model is used for power flow studies, contingency screening and to derive the sensitivity matrix (PTDF), which are calculated using MATPOWER (Zimmerman et al (2011)).

The market coupling problem for each market time unit (e.g. hour) can be formulated as a linear optimization problem with the objective of maximizing social welfare given by Eqs. 1-13 in Appendix 1. 
Table 2 Flow based parameter selection for the scenario calculations

\begin{tabular}{lll}
\hline Scenario & FB region & $\operatorname{minRAM}$ \\
\hline NTC & - & - \\
FB1 & CWE & $20 \%$ \\
FB2 & CWE & $70 \%$ \\
FB3 & Core & $70 \%$ \\
\hline
\end{tabular}

\subsection{Scenarios}

For the investigations, several scenarios for the year 2025 are used. Hence, the grid expansion state is a snapshot of 2025 including various expansion measures from the expansion plans. Figure 2 shows the transmission grid used in the study, where $380 \mathrm{kV}$ lines are shown in red, $220 \mathrm{kV}$ lines in green and HVDC lines in blue. The grid consists of 3,384 busses at 1,686 substations, with 6,680 AC lines and transformers, 24 HVDC lines (including interconnectors) and 344 reactive power compensation elements. The installed capacity for thermal power plants is chosen according to Platts (2016) following assumption on the economic lifetime of power plants and already known decommissioning decisions. The installed capacity of renewable energy sources is chosen according to the "Best Estimate" scenario for 2025 from ENTSO-E (2018). For the regional allocation, the generation profiles of renewable energies and temperature dependent load characteristics, weather data from the year 2016 have been used. To build a consistent scenario, the non-availability of conventional power plants correspond to the outages reported on the ENTSO-E transparency platform for the year 2016 (ENTSO-E (2021c)). The NTC values for the market simulations are chosen according to the ENTSO-E Mid-term Adequacy Forecast 2020 (ENTSO-E (2020b)), including the explicit profiles where given, but neglecting the stochastic unavailability. Also included are the external constraints for the Polish bidding zone. The market simulation is performed for 8,760 hours in weekly blocks. Storage optimization for pumped hydro storage power plants is performed for a weekly time horizon, where the state-of-charge is forced to $50 \%$ in the first and last time step, allowing for decoupling and calculation in parallel of the weekly blocks. Apart from Italy, which is modelled in two zones (Italy North and others), the market zones correspond to the bidding zones as they are present in the Day-Ahead market coupling. Reserves and strategic bids are omitted.

The different FB parameters for the experiments are given in Table 2. The scenario "NTC" represents the base case for the FB simulations and consists of a market simulation with NTC coupling constraints for the entire region. In scenario "FB1", FBMC is applied in the CWE region, with a minRAM criterion of $20 \%$ as it was in use in CWE until 2020. In scenario "FB2", FBMC is also applied in the
CWE region, but the minRAM level is increased to $70 \%$ as intended by the Clean Energy Package. The scenario "FB3" also applies a minRAM level of 70\%, but extends the geographic coverage of FBMC to the Core region. For all scenarios the PTDF threshold is chosen to 5\% and the CNEC threshold to $80 \%$ to ensure comparability.

\section{Results}

For the three FB scenarios, the change in electricity prices, the net export position and the binding line constraints are analyzed. The average electricity price is shown in Fig. 3. As stated in Sect. 3.2, Italy is modelled in two price zones. Nevertheless, all zones are depicted but the southern zones have the same price. The aggregated net export positions for the bidding zones in the three scenarios are shown in Fig. 4. The results for the scenario "NTC" are shown in Fig. 6 in Appendix 3.

The first comparison regards the impact of increasing the minimum capacity threshold (minRAM) for the CWE region. Keeping everything else constant, the net export positions, mean prices and binding grid constraints are compared for an increased minRAM level from $20 \%$ (FB1) to $70 \%$ in (FB2).

Most prominent is the change in the French bidding zone. The average electricity price increases by up to $11 \%$, due to an increased export position, which grows by $9 \%$. Prices in Belgium, Spain, the Netherlands and Portugal also show increased levels. In the southern Nordic zones prices decrease, which is induced by lower prices in Germany, without a significant change of net positions. The Baltic countries, Central and Eastern Europe and the Balkan zones also show reduced price levels compared to the $20 \%$ minRAM scenario.

The increased minRAM reduces the number of binding grid elements. This mostly affects intra-zonal constraints in Belgium and Germany, which are no longer in any hour restricting the market. This demonstrates the effectiveness of minimum trading capacities to reduce the importance for internal lines for the market clearing. Figure 5 shows that the majority of binding lines in the scenario with a minRAM of $70 \%$ are inter-connectors or adjacent elements. Another important result is the share of constraints being replaced by the minRAM condition. The minRAM share in Table 3 is indicating the number of CNECs that are subject to minRAM, i.e. where the available capacity for trading would be lower than allowed by the regulator. As anticipated, the number of replaced constraints increases from $16 \%$ in "FB1" to more than $50 \%$ with a higher minRAM in "FB2".

The difference between scenarios "FB2" and "FB3" is the extension of FBMC from CWE to the Core region, then 


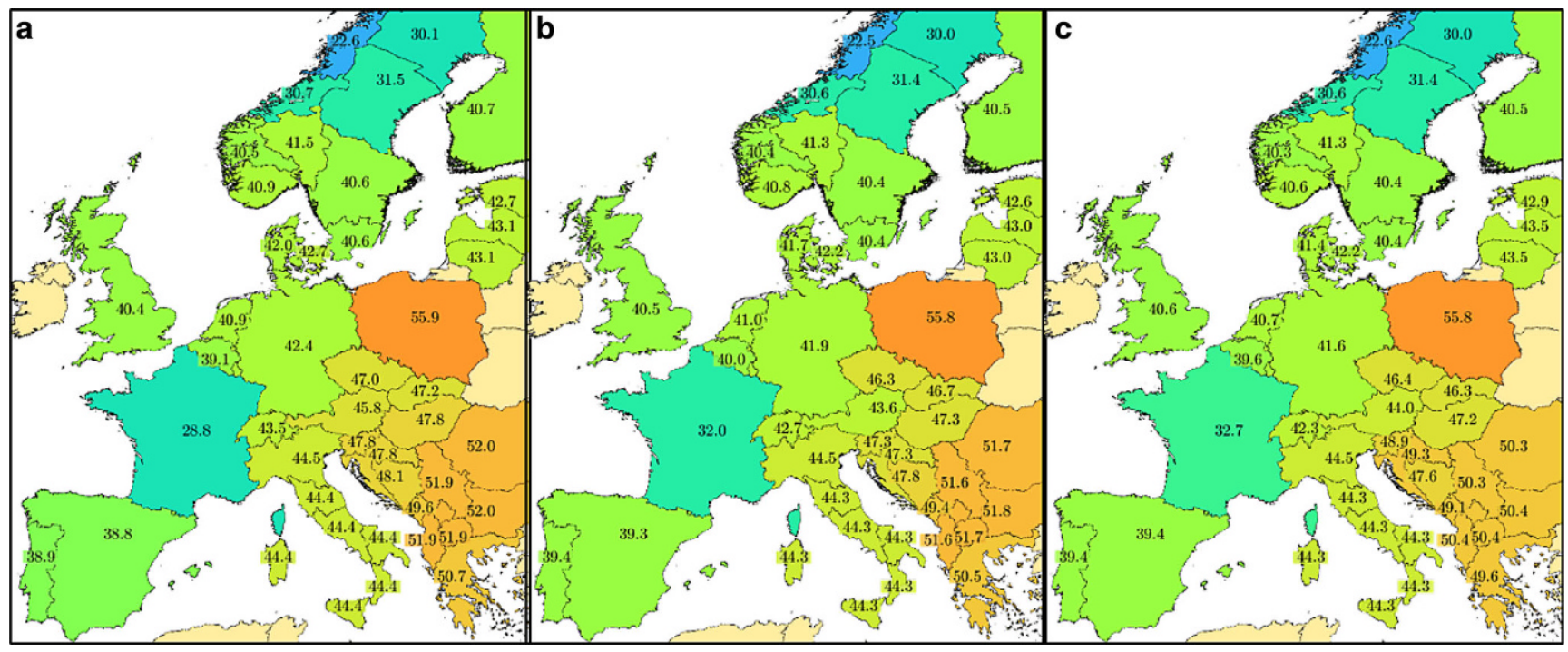

Fig. 3 Average Price in $€ / M W h$ for each bidding zone in the scenarios FB1 (a), FB2 (b) and FB3 (c)

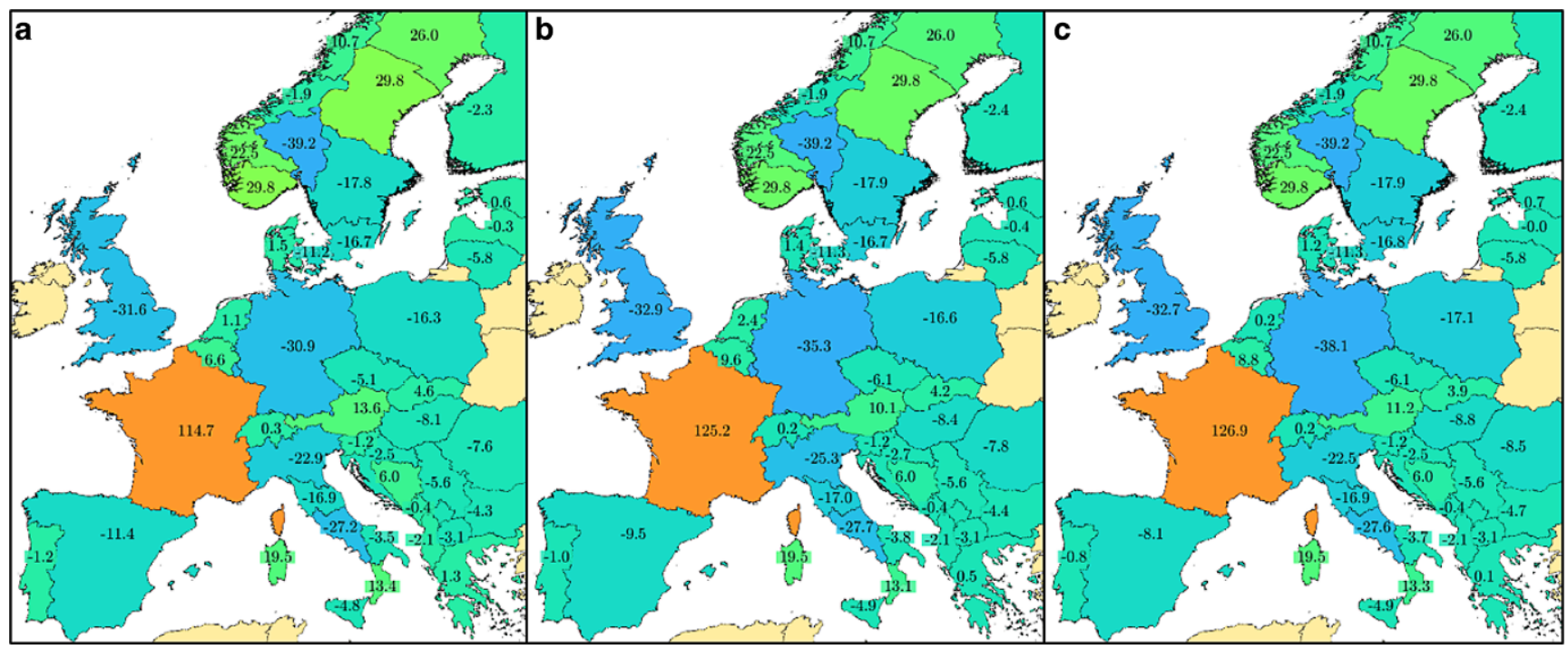

Fig. 4 Net export position for each bidding zone in TWh in the scenarios FB1 (a), FB2 (b) and FB3 (c)

covering a major part of the coupled European electricity markets in scenario "FB3". For bidding zones in Western Europe the effects are similar to the higher level of minRAM in CWE, with further increasing prices in France, Great Britain and Spain. Consumers in Romania and Hungary profit the most with regard to price levels. Despite not being part of the FB region, the southeastern European zones also show reduced price levels. Polish prices and net positions are mainly determined by the external import constraint, which makes the results comparably stable. The Nordic zones are almost not affected. Prices in the Baltic zones and some Central European zones increase slightly, making it difficult to identify a general trend. Interestingly, countries connected through NTCs to the FB region show relatively constant results across the investigated scenarios.
This indicates that, while the distribution among members of the FB region changes, the fundamental exchange patterns from and to the FB region remain unchanged in the calculations. For the binding grid elements, the effect is also similar to the observations in the CWE scenarios. Increased levels of minRAM reduce the number of binding constraints and push considered grid elements towards the inter-connectors, which are heavily impacted by cross-border flows. The total number of constraints is highly sensitive to the extension of the flow based region from CWE to Core as shown in the third column of Table 3. As each line generates two constraints (one for the flow in each direction), the number of RAM is twice the number of relevant network elements. The total number of RAMs in the model varies between 16.7 million for CWE and 28.3 million for the Core 
Fig. 5 Number of hours when CNECs are binding for different levels of minRAM; $20 \%$ in scenario FB1 (a) and $70 \%$ in scenario FB2 (b) for FBMC applied in the CWE region

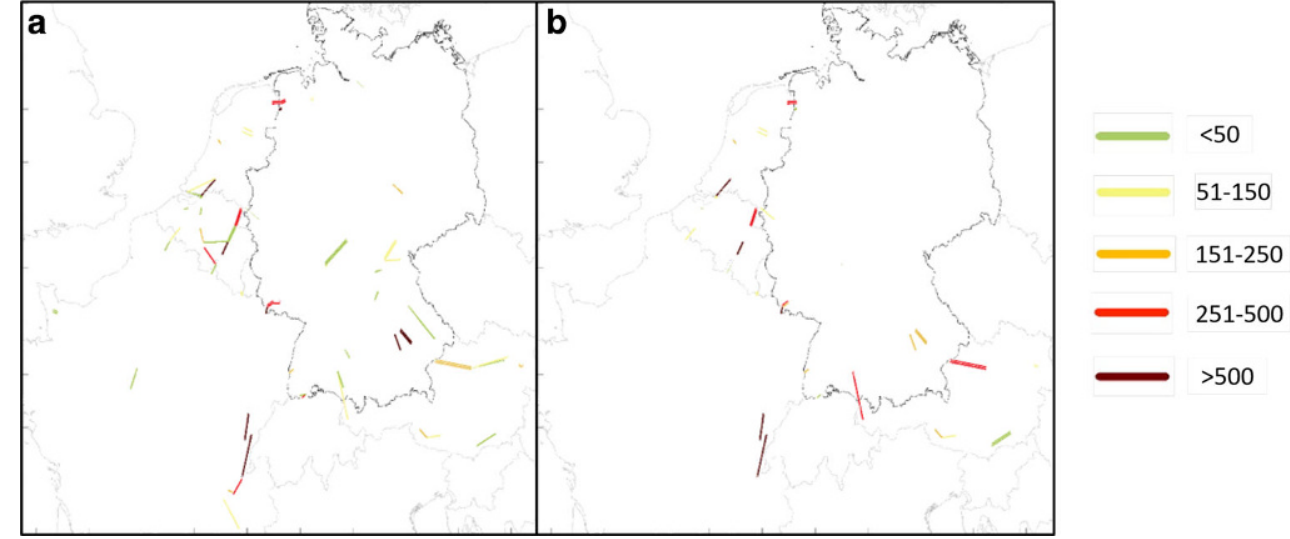

region. The number of CNECs also varies widely between hours, indicating the different utilization of the grid in different situations. The minimum number of CNEC in one hour over all scenarios is 996, while the maximum occurs in the Core scenario consisting of almost 15,600 constraints in a single hour.

The highly volatile number of constraints indicates that the possible exchange might be limited differently in changing grid conditions, i.e. they depend on the supply and load distribution in the grid. This is increasingly important in energy systems with high shares of RES, where supply depends upon volatile weather conditions and demand is more and more flexible due to new and flexible consumers. This makes the suitability of static NTCs, as they are often applied in scenario-based analysis of the energy system, highly questionable. Besides the aggregated implications presented here, this might result in more frequently changing operational patterns for power plants, with implications for life-time and flexibility needs, which should be addressed in further research.

Compared to the most restrictive scenario "FB1", which resembles FBMC in CWE until 2020 and is already found to increase transmission levels compared with the NTC market coupling, which was in operation beforehand ( CWE TSOs (2015)), the target scenario for the European internal market (FB3) with FBMC introduced in the Core region and a minRAM of $70 \%$ shows decisive differences with regard to prices and net export positions, both most notably visible in France. The difference in average price is up to $13 \%$, indicating increased export of relatively cheap nuclear energy. Indeed this impression is confirmed by a difference in net position of $10 \%$ between the two scenarios.

A last remark regards the difference between the FB results and the NTC market outcome, where a surprisingly large discrepancy is visible. French price levels and net positions in the scenario "NTC" are most closely resembled by the Core scenario with a minRAM of $70 \%$ (FB3). For possible reasons and implications, see Sect. 5.

\section{Critical review and wider implications}

Although the model accounts for the most important aspects of the market coupling process to assess the differences in parameter choice, the presented approach is not without limitations. The biggest limitation stems from the transmission network model. The model is a static representation for the entire calculated year. Moreover, the topology is reduced to one bus bar per voltage level and substation. This reduces the ability to account for topology adjustments carried out by the grid operators.

Furthermore, the considered contingencies only consist of line and transformer outages. Generator outages or failures of HVDC lines as well as contingencies of multiple elements are not considered. However, comparing the FBMC results with the NTC outcome, the results of the power flow studies seem to be rather too restrictive in the FB cases. Additional constraints from more sophisticated outage consideration would restrict the FB domain even further.

The power plant outages are modelled according to the historic 2016 outages to be consistent with the weather year. While this helps to account for e.g. low river levels and resulting non-availability, no data is included for power plants commissioned after 2016. In the considered scenario year, this leads to underproduction for units, which showed above average outages in 2016, while overestimating the production of plants without any modelled outages. On the aggregated bidding zone level, these effects can assumed to be minor.

Uncertainties, with regard to load, wind and solar generation as well as outages are neglected in the model, which uses perfect foresight. Also not accounted for are the intraday and reserve markets. While the the impact of reserves is neglected, the approach partly accounts for the intraday price effects, by clearing the entire electricity demand in the Day-Ahead time frame.

Finally, the calculations show that the FB scenarios mostly show lower exchanges than the NTC reference 
Table 3 Numerical Scenario data

\begin{tabular}{lllll}
\hline Scenario & minRAM share & \#RAM & $\max$ \#RAM/h & min\#RAM/h \\
\hline NTC & - & - & - & - \\
FB1 & $16 \%$ & $16,699,166$ & 14,050 & 996 \\
FB2 & $54 \%$ & $16,699,166$ & 14,050 & 996 \\
FB3 & $44 \%$ & $28,323,080$ & 15,596 & 1,718 \\
\hline
\end{tabular}

case, especially with regard to the French net position. This is an effect already reported by Marjanovic et al (2018), who attribute it to a misfit of the NTCs and the grid scenario. Apart from this, there are two possible explanations for this supposed contradiction. One is the conservative operating voltage assumed, when calculating the flow limits of the lines. These are based on 380 and $220 \mathrm{kV}$ respectively, while in reality many lines are operated above nominal voltage. An increase of $10 \%$ would equally increase line limits and allow for larger exchanges.

Looking at the CNEC loading graphs in Fig. 5, a relatively large number of lines in Belgium are binding. The grid operator in Belgium, is already applying Dynamic Line Rating (DLR) in the CWE FBMC and is expected to use this tool intensively in the future. DLR allows for dynamically allocating lower or higher flow limits on particular lines, depending on the current weather conditions (radiation, temperature, wind speed). This allows for potentially higher flows on the lines, especially in hours where large amounts of wind energy are fed into the grid, in contrast to the static ratings applied in the presented approach throughout the year. Finally, the assumptions under which the NTCs for the scenario were calculated are not known do the author. This might affect the eastern European regions, where the exchanges are systematically overestimated, which might be due to missing minRAM consideration in the NTC calculation for these regions.

The analysis results in three key insights:

1. Increased minimum trading capacities lead to more exchange, hence more base load capacity can be used. This usually consists of nuclear or lignite power plants. In the analysed scenarios, the strongest impact on the European energy mix is the increased French nuclear position, where larger trading capacities allow for additional output of up to $10 \mathrm{TWh}$. To put this into perspective, when comparing a scenario with or without minRAM, the difference in $\mathrm{CO} 2$ free generation could amount to around 100 TW h over the course of 10 years (around $15 \%$ of annual electricity demand in Germany today), which might have significant implications when evaluating the scenario.

2. Increased minRAM also reduces the amount of binding grid elements, which are shifted towards the inter-connectors. In this context, it might be tempting to consider market coupling approaches sufficient, which exclusively use inter-connector capacities in whichever form for market coupling. However, the intra-zonal distribution of flows, which results from the geospatially referenced supply and demand sources has a decisive impact on the FB domain, as shown by the changing number of binding constraints in the different hours of the year. Due to the more and more prominent effect of RES in the system, dynamic market coupling constraints, which consider the specific grid situation, have to be taken into account to represent the energy system adequately.

3. When analysing the effect of extending the FBMC region from CWE to Core, or in general comparing FBMC against NTC market coupling, care has to be taken concerning the NTC values, which are applied in the comparison. Poorly chosen NTCs or values inconsistent with the grid expansion state, make the comparison difficult and could lead to unintuitive results. This is also confirmed by Marjanovic et al (2018).

\section{Conclusion and Outlook}

In this article, a framework to model Flow Based Market Coupling (FBMC) for the internal European Electricity Market is presented. The developed framework is used to study the impact of different minimum transfer capacities (minRAM) for FBMC and compare its application in the Central Western Europe and the extended Core region, where FBMC is going to be introduced in the near future. FBMC is the target method for market coupling in Europe, but not considered in many studies, which analyse future scenarios of the European energy system. When working with such system studies, practitioners should be aware of the differences the market coupling method brings along and the implications for the market results. The presented results show that larger minRAM increase price convergence in the flow based region, while also reducing the number of relevant constraints for the market coupling process. Larger minRAM reduce the number of binding constraints in the market outcome, especially the number of internal grid elements as it is intended by the introduction of minRAM. This allows for more base load generation across Europe, which also affects emissions depending on the composition of the power plant fleet. In particular, the difference in minRAM level and geographic scope of the area, where FBMC is applied can account for price 
differences of up to $13 \%$. In the specific scenario analysed, the increased base load generation mainly consists of emission free nuclear output in France, which increases by up to $10 \mathrm{TWh}$. This clearly highlights the important role, which exchange capacities play in energy system scenarios. Due to changing utilization patterns in the grid because of weather dependant generation from renewable sources, dynamic market coupling constraints need to be considered instead of static values for net transfer capacities (NTC) in order to adequately represent the energy system. For the comparison of NTC and FBMC in future scenarios, care has to be taken with regard to NTC selection, as they have to represent a grid expansion state consistent with the scenario analysed. With regard to future work, the insights from the literature that welfare implications need to be considered across the entire congestion management process, should be investigated in the Core region, to quantify shifts in welfare distribution between FBMC and NTC and the implications of minRAM on the grid operation and congestion management. Finally, future research should focus on the integration of dynamic line rating into Flow Based Market Coupling and the economic effects, as this methodology promises enormous potential for increased flow limits in the majority of market situations.

Open Access This article is licensed under a Creative Commons Attribution 4.0 International License, which permits use, sharing, adaptation, distribution and reproduction in any medium or format, as long as you give appropriate credit to the original author(s) and the source, provide a link to the Creative Commons licence, and indicate if changes were made. The images or other third party material in this article are included in the article's Creative Commons licence, unless indicated otherwise in a credit line to the material. If material is not included in the article's Creative Commons licence and your intended use is not permitted by statutory regulation or exceeds the permitted use, you will need to obtain permission directly from the copyright holder. To view a copy of this licence, visit http://creativecommons.org/licenses/by/4. $0 /$.

\section{Appendix}

\section{Market Model Formulation}

The market coupling problem for each market time unit (e.g. hour) can be formulated as a linear optimization problem with the objective of maximizing social welfare given by Eqs. 1-13.

$$
\max _{p, q} W=\max _{p, q} \sum_{k=1}^{Z} \sum_{i=1}^{N_{d}^{k}} q_{i}^{d, k} p_{i}^{d, k}-\sum_{k=1}^{Z} \sum_{j=1}^{N_{s}^{k}} q_{j}^{s, k} p_{j}^{s, k}
$$

$$
\begin{aligned}
& \sum_{j=1}^{N_{s}^{k}} q_{j}^{s, k}-\sum_{i=1}^{N_{d}^{k}} q_{i}^{d, k}-\sum_{l=1}^{Z} t_{k l}+\sum_{l=1}^{Z} t_{l k}+N P_{k \in F B}=0, \\
& \forall k=1, \ldots, Z \\
& 0 \leq t_{k l} \leq N T C_{k l}, \quad \forall k, l=1, \ldots, Z \\
& 0 \leq \sum_{l \in C} t_{k l} \leq N T C_{k, C}^{\mathrm{Set}}, \quad \forall k=1, \ldots, Z \\
& R A M_{j}^{-} \leq \sum_{k \in F B} z P T D F_{j, k} N P_{k} \leq R A M_{j}^{+}, \\
& \forall j=1, \ldots, n_{C N E C} \\
& \sum_{k \in F B} N P_{k}=0 \\
& S O C_{i, t-1}+\eta_{i}^{\text {ch }} p_{i}^{\text {ch }}-\frac{p_{i}^{\text {dch }}}{\eta_{i}^{\text {dch }}}+i n f l o w_{i, t}=S O C_{i, t} \\
& \forall i=1, \ldots, n_{\text {storage }}, \forall t=1, \ldots, 8,760 \\
& q_{i}^{\min , d, k} \leq q_{i}^{d, k} \leq q_{i}^{\max , d, k} \\
& q_{j}^{\min , s, k} \leq q_{j}^{s, k} \leq q_{j}^{\max , s, k} \\
& p_{i}^{\min , \mathrm{ch}} \leq p_{i}^{\mathrm{ch}} \leq p_{i}^{\max , \mathrm{ch}} \\
& p_{i}^{\min , \mathrm{dch}} \leq p_{i}^{\mathrm{dch}} \leq p_{i}^{\max , \mathrm{dch}} \\
& N P_{k}^{\min } \leq N P_{k} \leq N P_{k}^{\max } \\
& S O C_{i}^{\text {min }} \leq S O C_{i, t} \leq S O C_{i}^{\max } \quad \forall t=1, \ldots, 8,760
\end{aligned}
$$

Where $\left(q_{i}^{d, k}, p_{i}^{d, k}\right)$ is the $i^{\text {th }}$ offer in the demand curve of the bidding zone $k$, consisting of quantity $q$ and price $p$, $\left(q_{j}^{s, k}, p_{j}^{s, k}\right)$ is the $j^{\text {th }}$ offer in the supply curve of the bidding zone $k$, consisting of quantity $q$ and price $p$ and $t_{k l}$ is the transit from bidding zone $k$ to bidding zone $l . N_{d}^{k}$ is the number of demand offers in zone $k, N_{s}^{k}$ is the number of supply offers in zone $k$ and $Z$ is the number of zones. $F B$ is the set of zones where FBMC is applied, NTC is the Net transfer capacity between zones, and NTCk, $C$ Set is the transfer limit from zone k to zones $l$ in a set $C$ where coordinated NTCs are applied. CNEC is the critical network elements including those in contingency cases, $R A M$ the remaining available margin in the two flow direction on each critical network element, $z P T D F$ is the zonal Power Transfer Distribution Factor which approximates the linear flow distribution under the assumptions of the Generation Shift Keys on the CNECs and NP are the net export position of the respective zone. $p_{i}^{\mathrm{ch}}$ and $p_{i}^{\mathrm{ch}}$ are the charging and discharging quantity respectively of storage unit $i$ in timestep $t$, which reflect the bidding quantities of storage units in Eqs. 1 and 2. SOC is the state of charge of storage units, $\eta_{i}^{\text {ch }}, \eta_{i}^{\text {dch }}$ the efficiency of charging and discharging respectively of storage unit $i$, inflow is the external inflow into storages. 


\section{Nomenclatura}

\begin{tabular}{|c|c|}
\hline $\mathrm{AC}$ & Alternating Current \\
\hline $\mathrm{CNE}$ & Critical Network Element \\
\hline CNEC & Critical Network Element under a Contingency \\
\hline CWE & $\begin{array}{l}\text { Central Western Europe, Capacity Calculation } \\
\text { Region }\end{array}$ \\
\hline CEE & $\begin{array}{l}\text { Central Eastern Europe, Capacity Calculation } \\
\text { Region }\end{array}$ \\
\hline ENTSO-E & $\begin{array}{l}\text { European association for the cooperation of } \\
\text { transmission system operators for electricity }\end{array}$ \\
\hline FB & flow based \\
\hline FBMC & Flow Based Market Coupling \\
\hline GSK & Generation Shift Key \\
\hline $\mathrm{kV}$ & kilo Volt \\
\hline NTC & Net Transfer Capacities \\
\hline NP & Net export position \\
\hline PTDF & Power Transfer Distribution Factor \\
\hline RAM & Remaining Available Margin \\
\hline $\min R A M$ & $\begin{array}{l}\text { minimum threshold for Remaining Available } \\
\text { Margin }\end{array}$ \\
\hline MIQP & Mixed-Integer Quadratic Program \\
\hline HVDC & High Voltage Direct Current \\
\hline RES & Renewable energy source \\
\hline TWh & Terawatt hour \\
\hline
\end{tabular}

\section{Additional results-Scenario "NTC"}

(Fig. 6)

Fig. 6 Results for scenario "NTC". Net position in TWh (a) and Average Price in $€ / M W h$ for each bidding zone

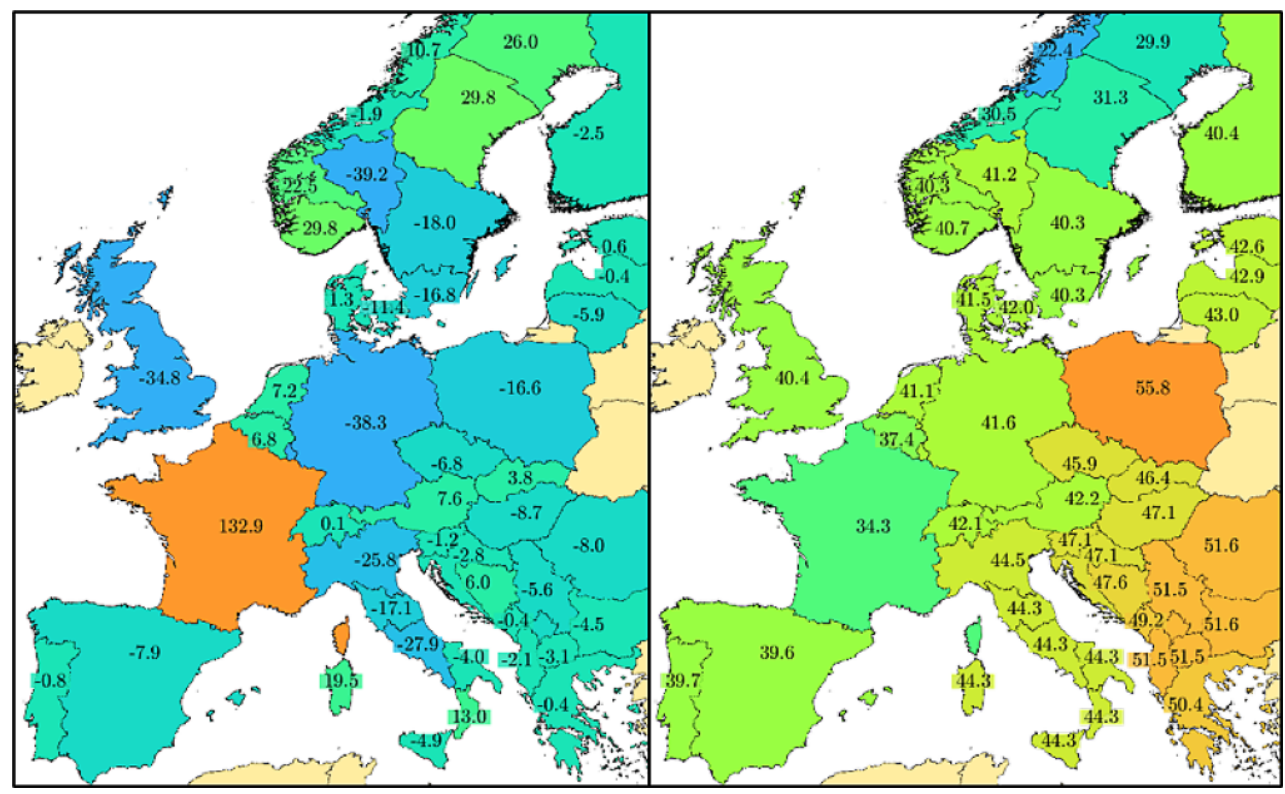


Acknowledgements Financial support was granted to the corresponding author through the cooperative doctoral program "Energy and Resource Efficiency" (ENRES) funded by the Federal State of BadenWuerttemberg.

Funding Open Access funding enabled and organized by Projekt DEAL.

Conflict of interest The authors declare that they have no conflict of interest.

\section{References}

Bjorndal E, Bjorndal MH, Cai H (2018) Flow-based market coupling in the european electricity market-A comparison of efficiency and feasibility. SSRN Journal. https://doi.org/10.2139/ssrn.3272188

Bo AH, Viken Kallset V, Oleinikova I, Farahmand H, Refsnas KL (2020) The impact of flow-based market coupling on the nordic region. In: 2020 17th International Conference on the European Energy Market (EEM), IEEE, pp 1-6 https://doi.org/10.1109/ EEM49802.2020.9221952

Byers C, Hug G (2020) Modeling flow-based market coupling: Base case, redispatch, and unit commitment matter. In: 2020 17th International Conference on the European Energy Market (EEM), IEEE, pp 1-6 https://doi.org/10.1109/EEM49802.2020.9221922

Carlini EM, Coluzzi C, Lanzellotto F, Pascucci A, Monopoli F, Ilea V, Bovo C, Le HL (2020) Simulation of euphemia's market coupling using a flow-based model for the region italy north. In: 2020 AEIT International Annual Conference (AEIT), IEEE, pp 1-6 https:// doi.org/10.23919/AEIT50178.2020.9241090

CREG (2017) Creg proposal for the adaption of the cbco selection method and the base case definition in the cwe flow based market coupling. https://www.creg.be/sites/default/files/assets/ Publications/Studies/F1687annex1.pdf. Accessed: 08 Oct 2021

CWE TSOs (2015) Cwe flow based market-coupling project: Parallel run performance report. https://www.jao.eu/sites/default/files/ 2020-04/Parallel\%20Run\%20performance\%20report\%2026-052015.pdf. Accessed: 08 Oct 2021

Dierstein C (2017) Impact of generation shift key determination on flow based market coupling. In: 2017 14th International Conference on the European Energy Market (EEM), IEEE, pp 1-7 https://doi.org/10.1109/EEM.2017.7981901

ENTSO-E (2016) Input grid datasets for the preparation of the tyndp 2016. https://www.entsoe.eu/publications/statistics-and-data/\# entso-e-on-line-application-portal-for-network-datasets. Accessed: 08 Oct 2021

ENTSO-E (2018) Tyndp 2018: Europe's network development plan to 2025, 2030 and 2040. https://tyndp.entsoe.eu/tyndp2018/ projects/. Accessed: 08 Oct 2021

ENTSO-E (2020a) Core flow based market coupling. https://www. entsoe.eu/news/2020/03/04/the-core-flow-based-market-couplingproject-parties-provide-an-update-on-the-project-planning-andinform-about-communication-channels-towardsmarket-participants/. Accessed: 08 Oct 2021

ENTSO-E (2020b) Maf 2020: Net transfer capacities. https://eepublicdownloads.entsoe.eu/clean-documents/ sdc-documents/MAF/2020/Net\%20Transfer\%20Capacities.7z. Accessed: 08 Oct 2021

ENTSO-E (2021) Entso-e transmission system map. https://www. entsoe.eu/data/map/

ENTSO-E (2021) Single day-ahead coupling (sdac). https://www. entsoe.eu/network_codes/cacm/implementation/sdac/\#what-doessdac-do. Accessed: 08 Oct 2021

ENTSO-E (2021) Unavailability of production and generation units. https://transparency.entsoe.eu/outage-domain/r2/unavailability OfProductionAndGenerationUnits/show. Accessed: 08 Oct 2021
European Commission (2019) Regulation (eu) 2019/943 of the european parliament and of the council: Rg2019/943. https:// eur-lex.europa.eu/legal-content/EN/TXT/HTML/?uri=CELEX: 32019R0943\&from $=$ EN. Accessed: 08 Oct 2021

Felling T, Felten B, Osinski P, Weber C (2019) Flow-based market coupling revised - part ii: Assessing improved price zones in central western europe. SSRN Journal. https://doi.org/10.2139/ssrn. 3404046

Felten B, Felling T, Osinski P, Weber C (2019) Flow-based market coupling revised - part i: Analyses of small- and large-scale systems. SSRN Journal. https://doi.org/10.2139/ssrn.3404044

Felten B, Osinski P, Felling T, Weber C (2021) The flow-based market coupling domain - why we can't get it right. Util Policy 70:101136. https://doi.org/10.1016/j.jup.2020.101136

Finck R, Ardone A, Fichtner W (2018) Impact of flow-based market coupling on generator dispatch in cee region. In: 2018 15th International Conference on the European Energy Market (EEM), IEEE, pp 1-5 https://doi.org/10.1109/EEM.2018.8469927

Hagspiel S, Jägemann C, Lindenberger D, Brown T, Cherevatskiy S, Tröster E (2014) Cost-optimal power system extension under flow-based market coupling. Energy 66:654-666. https://doi.org/ 10.1016/j.energy.2014.01.025

Henneaux P, Lamprinakos P, de Maere d'Aertrycke G, Karoui K (2021) Impact assessment of a minimum threshold on cross-zonal capacity in a flow-based market. Electr Power Syst Res 190:106693. https://doi.org/10.1016/j.epsr.2020.106693

Kristiansen T (2020) The flow based market coupling arrangement in europe: Implications for traders. Energy Strategy Rev 27:100444. https://doi.org/10.1016/j.esr.2019.100444

Kurzidem M (2010) Analysis of flow-based market coupling in oligopolistic power markets. PhD thesis, ETH Zurich, https:// doi.org/10.3929/ethz-a-006097327

Lang LM, Dallinger B, Lettner G (2020) The meaning of flow-based market coupling on redispatch measures in austria. Energy Policy 136:111061. https://doi.org/10.1016/j.enpol.2019.111061

Makrygiorgou DI, Andriopoulos NL, Dikaiakos C, Papaioannou GP (2020) Cross-border trading via market coupling in south east europe region. In: 2020 17th International Conference on the European Energy Market (EEM), IEEE, pp 1-6 https://doi.org/10. 1109/EEM49802.2020.9221995

Marien A, Luickx P, Tirez A, Woitrin D (2013) Importance of design parameters on flowbased market coupling implementation. In: 2013 10th International Conference on the European Energy Market (EEM), IEEE, pp 1-8 https://doi.org/10.1109/EEM.2013. 6607298

Marjanovic I, vom Stein D, van Bracht N, Moser A (2018) Impact of an enlargement of the flow based region in continental europe. In: 2018 15th International Conference on the European Energy Market (EEM), IEEE, pp 1-5 https://doi.org/10.1109/EEM.2018. 8470008

Matthes B, Spieker C, Rehtanz C (2017) Flow-based parameter determination in large-scale electric power transmission systems. In: 2017 IEEE Manchester PowerTech, IEEE, pp 1-6 https://doi.org/ 10.1109/PTC.2017.7981216

Matthes B, Spieker C, Klein D, Rehtanz C (2019) Impact of a minimum remaining available margin adjustment in flow-based market coupling. In: 2019 IEEE Milan PowerTech, IEEE, pp 1-6 https://doi.org/10.1109/PTC.2019.8810504

Mekonnen MT, Belmans R (2012) The influence of phase shifting transformers on the results of flow-based market coupling. In: 2012 9th International Conference on the European Energy Market, IEEE, pp 1-7 https://doi.org/10.1109/EEM.2012.6254746

Next Kraftwerke (2021) What is market coupling? https://www.nextkraftwerke.com/knowledge/market-coupling. Accessed: 08 Oct 2021

Platts (2016) Platts world electric power plants database

Poplavskaya K, Totschnig G, Leimgruber F, Doorman G, Etienne G, de Vries L (2020) Integration of day-ahead market and redispatch to 
increase cross-border exchanges in the european electricity market. Appl Energy 278:115669. https://doi.org/10.1016/j.apenergy. 2020.115669

Ruppert M, Slednev V, Finck R, Ardone A, Fichtner W (2020) Utilising distributed flexibilities in the european transmission grid. In: Bertsch V, Ardone A, Suriyah M, Fichtner W, Leibfried T, Heuveline V (eds) Advances in energy system optimization, trends in mathematics. Springer, Cham, pp 81-101 https://doi.org/10.1007/ 978-3-030-32157-4_6

Schavemaker PH, Beune RJ (2013) Flow-based market coupling and bidding zone delimitation: Key ingredients for an efficient capacity allocation in a zonal system. In: 2013 10th International Conference on the European Energy Market (EEM), IEEE, pp 1-6 https://doi.org/10.1109/EEM.2013.6607366

Schönheit D, Weinhold R, Dierstein C (2020) The impact of different strategies for generation shift keys (gsks) on the flow-based market coupling domain: A model-based analysis of central western europe. Appl Energy 258:114067. https://doi.org/10.1016/j. apenergy.2019.114067

Schönheit D, Dierstein C, Möst D (2021) Do minimum trading capacities for the cross-zonal exchange of electricity lead to welfare losses? Energy Policy 149:112030. https://doi.org/10.1016/j. enpol.2020.112030

Schönheit D, Kenis M, Lorenz L, Möst D, Delarue E, Bruninx K (2021) Toward a fundamental understanding of flow-based market coupling for cross-border electricity trading. Adv Appl Energy 2:100027. https://doi.org/10.1016/j.adapen.2021.100027

Sebestyen M, Divenyi D, Sores P (2018) An enhanced calculation method of generation shift keys in flow based market coupling. In: 2018 15th International Conference on the European Energy Market (EEM), IEEE, pp 1-5 https://doi.org/10.1109/EEM.2018. 8469923

Slednev V, Ruppert M, Bertsch V, Fichtner W, Meyer-Hübner N, Suriyah M, Leibfried T, Gerstner P, Schick M, Heuveline V (2017) Regionalizing input data for generation and transmission expansion planning models. In: Bertsch V, Fichtner W, Heuveline $\mathrm{V}$, Leibfried $\mathrm{T}$ (eds) Advances in energy system optimization, trends in mathematics. Springer, Cham, pp 205-217 https://doi. org/10.1007/978-3-319-51795-7_13

Slednev V, Bertsch V, Ruppert M, Fichtner W (2018) Highly resolved optimal renewable allocation planning in power systems under consideration of dynamic grid topology. Comput Oper Res 96:281-293. https://doi.org/10.1016/j.cor.2017.12.008

Sores P, Divenyi D, Raisz D (2013) Flow-based capacity calculation method used in electricity market coupling. In: 2013 10th International Conference on the European Energy Market (EEM), IEEE, pp 1-7 https://doi.org/10.1109/EEM.2013.6607285

van den Bergh K, Boury J, Delarue E (2016) The flow-based market coupling in central western europe: Concepts and definitions. Electr J 29(1):24-29. https://doi.org/10.1016/j.tej.2015.12.004

Weinhold R (2021) Evaluating policy implications on the restrictiveness of flow-based market coupling with high shares of intermittent generation: A case study for central western europe. http:// arxiv.org/pdf/2109.04940v1. Accessed: 08 Oct 2021

Weinhold R, Mieth R (2021) Uncertainty-aware capacity allocation in flow-based market coupling. http://arxiv.org/pdf/2109.04968v2. Accessed: 08 Oct 2021

Wyrwoll L, Kollenda K, Muller C, Schnettler A (2018) Impact of flowbased market coupling parameters on european electricity markets. In: 2018 53rd International Universities Power Engineering Conference (UPEC), IEEE, pp 1-6 https://doi.org/10.1109/ UPEC.2018.8541904

Wyrwoll L, Blank A, Muller C, Puffer R (2019) Determination of preloading of transmission lines for flow-based market coupling. In: 2019 16th International Conference on the European Energy Market (EEM), IEEE, pp 1-6 https://doi.org/10.1109/EEM.2019. 8916565

Zad BB, Toubeau JF, Vatandoust B, Bruninx K, de Grève Z, Vallée F (2021) Enhanced integration of flow-based market coupling in short-term adequacy assessment. Electr Power Syst Res 201:107507. https://doi.org/10.1016/j.epsr.2021.107507

Zimmerman RD, Murillo-Sanchez CE, Thomas RJ (2011) Matpower: Steady-state operations, planning, and analysis tools for power systems research and education. IEEE Trans Power Syst 26(1):12-19. https://doi.org/10.1109/TPWRS.2010.2051168 